\title{
The Next NGS Challenge Conference: Data Processing and Integration
}
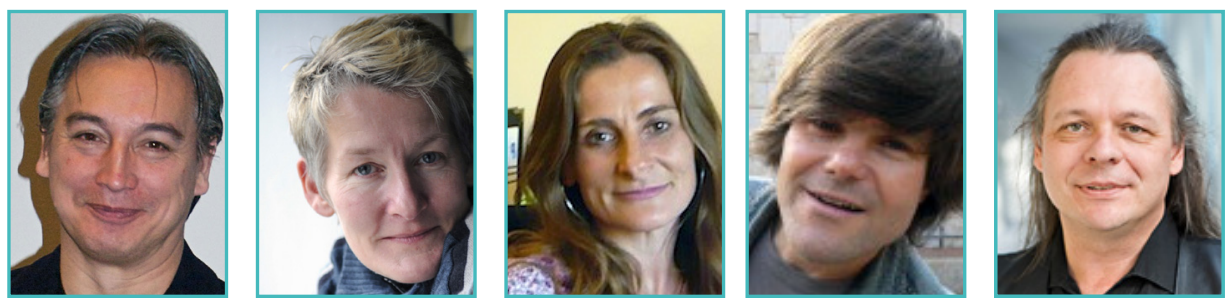

Erik Bongcam-Rudloff ${ }^{\circledR}$, Teresa K Attwood ${ }^{2}$, Ana Conesa ${ }^{3}$, Andreas Gisel ${ }^{4}$, Burkhard Ros ${ }^{5}$

'Swedish University of Agricultural Sciences, Uppsala, Sweden

2University of Manchester, Manchester, United Kingdom

${ }^{3}$ Bioinformatics and Genomics Department of the Centro de Investigación Príncipe Felipe, Valencia, Spain ${ }^{4} \mathrm{CNR}$, Institute for Biomedical Technologies, Bari, Italy

${ }^{5}$ Department for Bioinformatics and Computational Biology, Fakultät für Informatik, Garching, Germany

Next Generation Sequencing (NGS) is a highly parallelised approach for quickly and economically sequencing new genomes, re-sequencing large numbers of known genomes, or for rapidly investigating transcriptomes under different conditions. Producing data on an unprecedented scale, these techniques are now driving the generation of knowledge (especially in biomedicine and molecular life sciences) to new dimensions. The massive data volumes being generated by these new technologies require new datahandling and -storage methods. Hence, the Life Science community urgently needs new and improved approaches to facilitate NGS data management and analysis.

A "moving target", this field requires that bioinformaticians, computer scientists and biomedical scientists join their expertise to bring NGS data management and analysis to new levels of efficiency and integration.

In your hands, you hold the proceedings of the "Next NGS Challenge, Data Processing and Integration, Conference" organised during 1617 May 2013 in Valencia, Spain. This conference - a joint event of the EU COST Action BM1006, SeqAhead; the Global Bioinformatics Network, EMBnet; the FP7 Project, STATegra; and the International Society for Computational Biology, ISCB - aims to become a regular, dedicated meeting on cutting-edge NGS applications. As such, it will continue to bring together biologists, computational biologists and bioinformaticians to discuss new challenges in high-throughput sequencing, and to highlight new trends in NGSbased genome research.
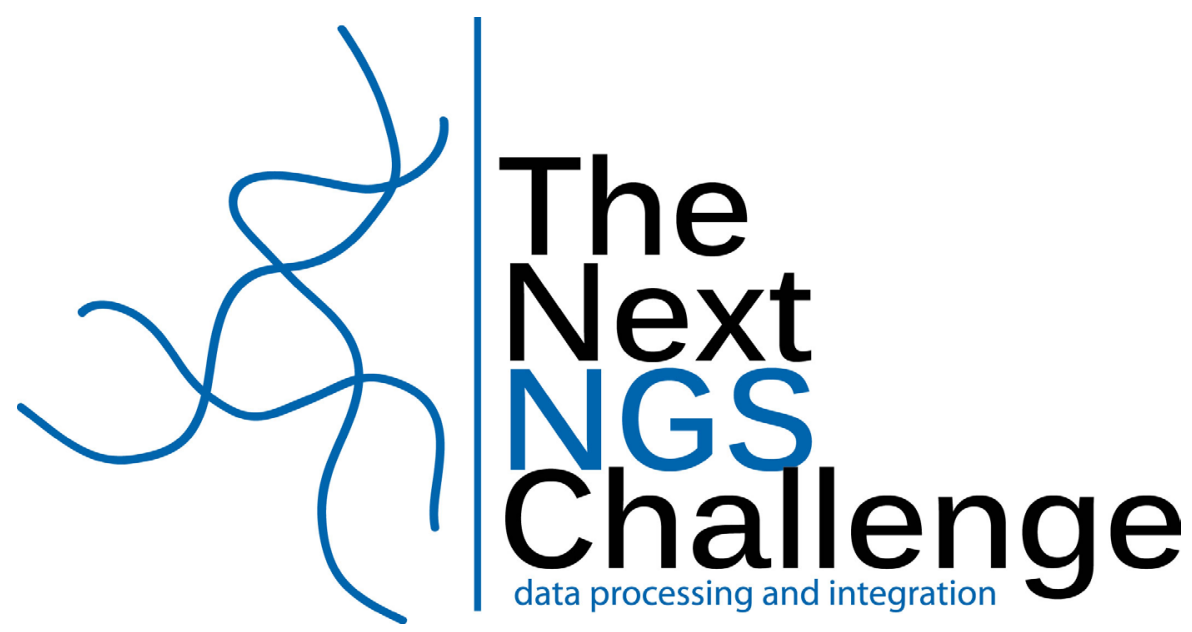